\title{
An enterohaemorrhagic Escherichia coli outbreak spread through the environment at an institute for people with intellectual disabilities in Japan in 2005
}

\author{
Masaki Ota, ${ }^{a}$ Taro Kamigaki, ${ }^{b}$ Satoshi Mimura, ${ }^{c}$ Kazutoshi Nakashima, ${ }^{d}$ and Takashi Ogami ${ }^{\mathrm{C}}$ \\ Correspondence to Masaki Ota (email: otam@jata.or.jp)
}

Objective: An enterohaemorrhagic Escherichia coli (EHEC) outbreak at an institute with multiple facilities for children and adults with intellectual disabilities was investigated to characterize the cases and identify risk factors for infection.

Methods: A case was defined as a resident, a staff member or a visitor at the institute from 16 May through 30 June 2005 testing positive for type 2 Vero toxin-producing EHEC 0157:H7 (confirmed case) or exhibiting bloody diarrhoea for two or more days (probable case). We collected and analysed demographic, clinical, laboratory and individual behaviour data to identify possible risk factors for infection and infection routes.

Results: We recorded 58 confirmed cases, of which 13 were symptomatic. One probable case was also found. The median age of the patients was 37 years (range: 6-59 years). Thirty-six patients (61\%) were male. Thirteen patients (93\%) had diarrhoea and six (43\%) had abdominal pain. Two developed haemolytic-uraemic syndrome but recovered. All the patients were treated with antibiotics and tested negative after treatment. Some residents had problems with personal hygiene. The residents of one of the facilities who cleaned a particular restroom had 18.0 times higher odds of being infected with EHEC (95\% confidence interval: 4.0-102.4) than those who did not.

Discussion: The source of the outbreak could not be identified; however, the infection may have spread through environmental sources contaminated with EHEC. We recommend that institutional settings, particularly those that accommodate people with intellectual disabilities, clean restrooms as often as possible to reduce possible infection from contact with infected surfaces.

$\mathrm{E}$ nterohaemorrhagic Escherichia coli (EHEC) was first reported in 1983 in the United States of America. ${ }^{1}$ Infection can cause diarrhoea, haemorrhagic colitis and haemolytic-uraemic syndrome (HUS). ${ }^{2}$ Outbreaks involving EHEC can be spread through infected food, ${ }^{2}$ water, ${ }^{3}$ direct contact with infected humans ${ }^{4}$ or animals ${ }^{5,6}$ or exposure to infected environments. ${ }^{7}$ In Japan, EHEC is a reportable communicable disease; the largest outbreak to date was associated with consumption of white radish sprouts affecting about 8400 schoolchildren in $1997 .{ }^{8}$

On 6 June 2005, a physician informed a local health office of Oita Prefecture in western Japan of two EHEC cases at an institute for adults and children with intellectual disabilities.
The objectives of the study were to characterize the epidemiology of the cases and identify possible risk factors for EHEC infection in this outbreak.

\section{METHODS}

A case was defined as a resident, a staff member or a participant in the activities at the institute for at least one day from 16 May through 30 June 2005 who had a stool specimen that tested positive for Vero toxin type 2 (VT2)producing EHEC 0157:H7 (confirmed case) or exhibited bloody diarrhoea for two days or more (probable case), considering the long incubation period (1 to 9 days) of EHEC.

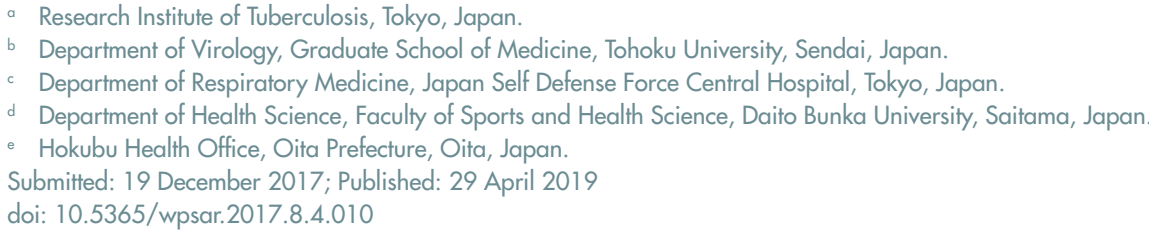


At the time of the outbreak, the institute had 162 long-term and 13 short-term residents, 20 participants of day care and vocational training and 81 staff members (total 276) in three facilities (Facilities A, B and C). Facilities $A$ and $B$ were for children and adults with intellectual disabilities, respectively, and Facility $\mathrm{C}$ was for vocational training and residence for adults with mild intellectual disabilities. Some residents of Facility A attended a school outside the facility run by another organization five days a week. Residents of Facility $\mathrm{C}$ worked outside the institute, and about 20 persons who lived outside the institute attended vocational training held at Facility $\mathrm{C}$.

To obtain relevant epidemiological and clinical information, the local health office staff and the authors interviewed the staff members at the time of the outbreak and reviewed charts of the residents and participants at the institute using semi-standardized instruments, to determine demographics (e.g. age, sex), symptoms and signs, date of onset and potential exposure history. Affected patients were referred to a local hospital where we also reviewed patient charts. Environmental samples, including surface swabs of doorknobs, water taps and stair and hand rails, were also collected from the institute by the local health office staff and examined at the Oita Prefecture Hygiene and Environment Centre (OPHEC). ${ }^{9}$ The local health office collected stool samples from residents, vocational training participants and staff of the school for the intellectually disabled. The samples were examined for EHEC at the local health office and OPHEC using the National Institute of Infectious Diseases' standard method. ${ }^{10}$ Staff members of the institute collected stool samples from the kitchen staff that were examined at a private laboratory. We randomly selected half of the strains isolated from the confirmed cases which were further analysed with pulsed-field gel electrophoresis (PFGE). ${ }^{11}$

We conducted a nested, unmatched case-control study of the residents, vocational training participants and the staff at each facility. For the case-control study, we defined a case as a person with bacteriologically confirmed EHEC infection. All residents at the facilities who tested negative for EHEC and did not exhibit diarrhoea between 1 and 9 June were chosen as controls. Individuals who had diarrhoea but tested negative for EHEC were not included in the case-control study. We conducted interviews with the patients and controls with the assistance of the staff, if necessary, using a structured questionnaire. Potential risk factors were age; sex; daily living skills, including personal hygiene (whether one was able to wash hands, brush teeth or bathe independently); toilet hygiene (whether one was able to defecate independently or one had allotriophagic behaviours); and needing assistance in taking meals; participating in day care or vocational training; and specific restrooms used or cleaned. Stratified analysis by Mantel-Haenszel method was employed to explore and adjust odds ratios if the univariable analysis revealed statistically significant result ( $p$-value $<0.05$ )

Statistical tests were conducted using $\mathrm{R}$ software (The R Foundation for Statistical Computing, Vienna, Austria), and a $p$-value of $<0.05$ was considered statistically significant.

\section{Ethics statement}

The investigation was conducted in accordance with the Infectious Disease Control Act of 1999 and the Food Safety Act of 1947 of Japan, which grants the prefectural health director the authority to collect epidemiological information and biologic specimens from patients without obtaining formal consent, in the event of an outbreak of certain confirmed or suspected communicable diseases, including EHEC.

\section{RESULTS}

All 276 residents, staff members, and participants of day care and vocational training had stool specimens collected and examined for EHEC. Fifty-nine cases were reported, of which $58(98 \%)$ were confirmed and one was probable (2\%). The probable case was a resident of Facility $\mathrm{C}$ who had continuous bloody diarrhoea but had been treated with antibiotics before the stool examination and tested negative for EHEC. Overall, 14 (24\%) cases were symptomatic. The median age of the cases was 37 years (range: $6-59$ years), and $36(61 \%)$ cases were male. Four staff members exhibited non-bloody diarrhoea during the outbreak period; all four tested negative for EHEC and were eventually determined not to be confirmed or probable cases.

Among the staff, there were five cases (6.2\%). No staff members were out ill before the first case report on 1 June. No children in the school, except for those who were residents of Facility A, tested positive for EHEC. 
Table 1. EHEC 0157:H7 positivity among staff, residents and day care participants of an institute for people with intellectual disabilities, Japan, 2005

\begin{tabular}{|c|c|c|c|c|c|}
\hline & & & \multicolumn{2}{|c|}{$\begin{array}{l}\text { Bacteriologically } \\
\text { positive }\end{array}$} & \multirow[b]{2}{*}{ Total } \\
\hline & & & $\mathrm{n}$ & $\%$ & \\
\hline \multirow[t]{7}{*}{ Facility A } & Residents & Male & 10 & 25.0 & 40 \\
\hline & & Female & 1 & 8.3 & 12 \\
\hline & Participants & Male & 0 & 0 & 5 \\
\hline & & Female & 0 & 0 & 4 \\
\hline & Staff & Male & 0 & 0 & 5 \\
\hline & & Female & 2 & 9.1 & 22 \\
\hline & Subtotal & & 13 & 14.8 & 88 \\
\hline \multirow[t]{7}{*}{ Facility B } & Residents & Male & 18 & 37.5 & 48 \\
\hline & & Female & 8 & 36.4 & 22 \\
\hline & Participants & Male & 0 & 0 & 2 \\
\hline & & Female & 0 & 0 & 0 \\
\hline & Staff & Male & 2 & 16.7 & 12 \\
\hline & & Female & 0 & 0 & 22 \\
\hline & Subtotal & & 28 & 26.4 & 106 \\
\hline \multirow[t]{7}{*}{ Facility C } & Residents & Male & 5 & 17.8 & 28 \\
\hline & & Female & 6 & 50.0 & 12 \\
\hline & Participants & Male & 1 & 6.7 & 15 \\
\hline & & Female & 4 & 57.1 & 7 \\
\hline & Staff & Male & 0 & 0 & 9 \\
\hline & & Female & 1 & 9.1 & 11 \\
\hline & Subtotal & & 17 & 20.7 & 82 \\
\hline Total & & & 58 & 21.0 & 276 \\
\hline
\end{tabular}

There were no reports of any diarrhoea among staff members and children at the school, except for those who were residents of Facility A.

The breakdown of bacteriological test results by facility is shown in Table 1 . When stratified by sex and location, the infection rate was highest in women residents of Facility C $(52.6 \%, 95 \%$ confidence interval $[\mathrm{Cl}]$ : $28.8-75.5 \%)$.

Fig. 1 shows the epidemic curve of the 14 symptomatic ( 13 confirmed and one probable) cases. Four of the five residents who exhibited symptoms and signs from 1 to 2 June participated in a vocational training held on the 1st floor of Facility $\mathrm{C}$; however, we were not able to identify a period of close contact or a possible event that may have transmitted EHEC among them during the training, since they participated in separate and different tasks. Three patients of Facility $\mathrm{C}$ are clustered on 7-8 June following the first case at the same facility on 1 June.

The predominant clinical symptoms and signs of the 14 symptomatic cases were diarrhoea (13/14 cases, $93 \%)$, including bloody diarrhoea (2,14\%); abdominal pain $(6,43 \%)$; nausea or vomiting $(3,22 \%)$; and fever $\geq 37.5{ }^{\circ} \mathrm{C}(1,7 \%)$. Two patients (14\%) developed HUS; however, both fully recovered. Of the 10 symptomatic patients $(71 \%)$ who sought medical care, five (36\%) were hospitalized. No residents or staff members died from this outbreak.

EHEC 0157:H7 (VT2) was detected in the stool of 58 case patients. PFGE analysis found that all of the 28 randomly selected case patients had nearly identical strains with differences within two bands. ${ }^{11}$ The strain was also later found in one patient in Nakatsu, about $100 \mathrm{~km}$ north-west of the institute in July 2005; however, an epidemiological investigation found no direct link between that case and cases detected in this outbreak.

All individuals with confirmed infection, including those who were asymptomatic, were treated with antibiotics and tested negative twice for EHEC 0157:H7 48 hours after the end of treatment. One patient with HUS was treated with fosfomycin, and the other was treated with a fluoroquinolone before onset of HUS. Of 45 asymptomatic individuals, 37 took fosfomycin, six levofloxacin and one each tosufloxacin and cefpodoxime proxetil.

We studied potential risk factors for EHEC 0157:H7 infection. Among the resident cases, $88 \%$ were independent with respect to feeding, and $54 \%$ were independent with respect to urinating. However, most residents required assistance with personal hygiene and grooming (assistance was required by $63 \%$ with hand washing, $67 \%$ with defecation, and $60 \%$ with brushing teeth). Some residents ( $16 \%$ of Facilities A and B) had problems with personal hygiene, in particular allotriophagic behaviour (i.e. eating one's own feces) and manipulating their own feces.

Eleven kitchen staff members, including three nutritionists, worked in a single kitchen preparing meals 
Fig. 1. Epidemic curve of symptomatic cases by date of symptom onset during an outbreak of EHEC 0157:H7 at an institute for people with intellectual disabilities, Japan, 2005

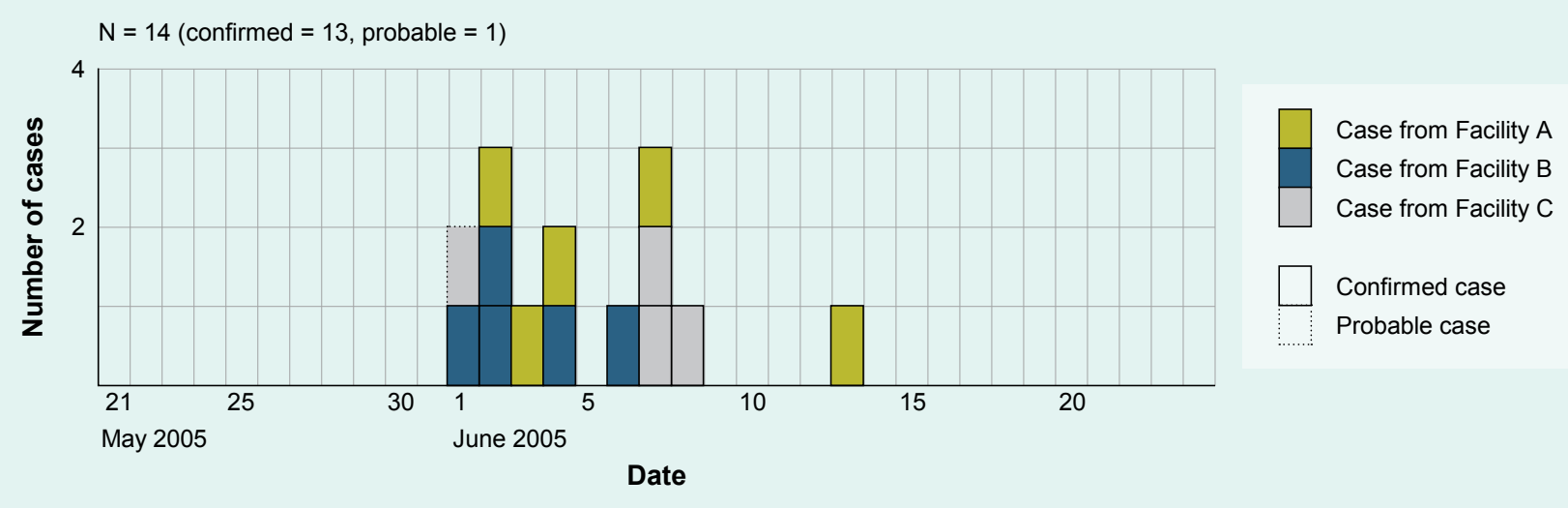

for the residents and staff who ate in dining halls in each facility. Food items served to the residents and staff were almost identical. Three nutritionists took turns eating each meal, and none of them exhibited diarrhoea during the outbreak period. All the kitchen staff tested negative for EHEC in three separate stool samples collected on 20 May (just before the outbreak as part of routine screening for food-handlers), on 3-4 June and 8-9 June. Samples of the meals provided from 23 to 30 May were stored in a freezer, and all samples tested negative for EHEC. Water samples from the tap water were all culture negative for EHEC. Chlorine levels of the tap water were checked and recorded every day and were consistently greater than $0.1 \mathrm{ppm}$.

We investigated the pre-outbreak routines for cleaning the toileting areas. The toilets of Facilities $A$ and $B$ were cleaned every day by the staff, two toilets on the 1st floor of Facility $C$ were cleaned every day by external vocational training participants and residents of Facility $\mathrm{C}$, and toilets on the 2nd and 3rd floors of Facility $\mathrm{C}$ were cleaned by the residents. The cleaning was normally done with detergent applied with reusable mops and cloths. Gloves were not always used during the cleaning before the outbreak. The time spent cleaning each restroom was normally 20 to 30 minutes.

No animals were brought into the facilities before or during the outbreak period.

Upon the recognition of the outbreak, the facilities introduced additional disease prevention and control measures, including encouraging intensive hand washing before meals and after toilet use, increasing monitoring of residents with allotriophagic behaviour, strengthening daily diarrhoea surveillance and cleaning surfaces three times per day. Prior to the outbreak, an infection control protocol was developed; anecdotally, the protocol was not followed consistently.

The results of the primary univariable analyses are shown in Table 2, and the stratified analyses for the residents of Facilities $A$ and $B$ are shown in Table 3. Additional univariable analyses are listed in Table S1. The univariable analysis found those residents who took meals at a certain table of Facility B were 9.7 (95\% $\mathrm{Cl}$ : 1.1-89.4) times the odds of being infected with EHEC (Table S1B). The inability to independently wash one's own hands was significantly associated with being a case (OR: $5.3,95 \% \mathrm{Cl}: 1.5-29.4$ ), specifically in men (OR: 12.9, 95\% Cl: 1.8-562.6) and not women (OR: 1.5, 95\% Cl: 0.2-18.7) (Table 3). Those who were unable to independently wash their own hands were more likely to be cases regardless of allotriophagic behavior (adjusted odds ratio [aOR]: $4.4,95 \% \mathrm{Cl}: 1.2-16.0$ ) or needing assistance in defecation (aOR: 4.3, 95\% Cl: 1.1-16.3). At Facility $\mathrm{C}$, it was found that the residents who had cleaned the female restroom on the 1st floor had 18.0 (95\% Cl: 4.0-104.4) times the odds of being infected with EHEC than those who did not (Table 2B).

\section{DISCUSSION}

We investigated an EHEC outbreak that occurred during 1-13 June 2005, affecting 59 residents and staff members of an institute for children and adults with 
Table 2. Individual characteristics associated with EHEC 0157:H7 infection in univariable analyses of an outbreak investigation at an institute for people with intellectual disabilities, Japan, 2005

\begin{tabular}{|c|c|c|c|c|}
\hline Individual risk factor & Cases (\%) & Controls (\%) & OR & $95 \% \mathrm{Cl}$ \\
\hline \multicolumn{5}{|c|}{ A. Residents of Facilities A and B ( 34 cases and 82 controls) } \\
\hline Female sex & $8(23.5)$ & $22(26.8)$ & 0.8 & $0.3-2.3$ \\
\hline Manipulates own faeces & $7(20.6)$ & $16(19.5)$ & 1.1 & $0.3-3.1$ \\
\hline Has habit of touching own buttocks & $6(17.6)$ & $8(9.8)$ & 2.0 & $0.5-7.2$ \\
\hline Has used a toilet outside the facility & $6(17.6)$ & $5(6.1)$ & 3.3 & $0.8-14.7$ \\
\hline Lies on the floor daily & $6(17.6)$ & $10(12.2)$ & 1.7 & $0.4-5.6$ \\
\hline Licks walls and tiles daily & $3(8.8)$ & $1(1.2)$ & 7.7 & $0.6-415.4$ \\
\hline Sucks one's fingers daily & $7(20.6)$ & $15(18.3)$ & 1.2 & $0.4-3.4$ \\
\hline Engages in allotriophagic behaviour & $10(29.4)$ & $9(11.0)$ & 3.3 & $1.1-10.5$ \\
\hline Loiters in the facility daily & $7(20.6)$ & $17(20.7)$ & 1.0 & $0.3-2.9$ \\
\hline Takes medication & $14(41.2)$ & $30(36.6)$ & 1.2 & $0.5-3.0$ \\
\hline Being unable to wash own hands & $31(91.2)$ & $54(65.9)$ & 5.3 & $1.5-29.0$ \\
\hline Being unable to take meals independently & $4(11.8)$ & $21(25.6)$ & 1.3 & $0.1-1.3$ \\
\hline Being unable to defecate independently & $25(73.5)$ & $48(58.5)$ & 2.0 & $0.8-5.4$ \\
\hline Needs assistance or direction in defecating & $16(47.1)$ & $21(25.6)$ & 2.6 & $1.0-6.4$ \\
\hline Needs assistance in bathing & $20(58.8)$ & $46(56.1)$ & 1.0 & $0.4-2.4$ \\
\hline In day care group $\mathrm{A} 1^{*}$ & $4(11.8)$ & $10(12.2)$ & 1.0 & $0.2-3.7$ \\
\hline In day care group $\mathrm{A} 2^{*}$ & $6(17.6)$ & $7(8.5)$ & 2.3 & $0.6-8.7$ \\
\hline In day care group $B 1^{*}$ & $5(14.7)$ & $6(7.3)$ & 2.2 & $0.5-9.3$ \\
\hline In day care group B2* & $3(17.6)$ & $7(8.5)$ & 1.0 & $1.0-4.9$ \\
\hline In day care group $\mathrm{S}^{*}$ & $3(17.6)$ & $7(8.5)$ & 1.0 & $1.0-4.9$ \\
\hline In day care group $\mathrm{V}^{*}$ & $5(14.7)$ & $5(6.1)$ & 2.6 & $0.6-12.2$ \\
\hline \multicolumn{5}{|c|}{ B. Residents, participants and staff members in activities of Facility C (18 cases and 65 controls) ${ }^{\star *}$} \\
\hline Female sex & $11(61.1)$ & $19(29.2)$ & 3.7 & $1.1-13.3$ \\
\hline Being a staff member & $1(5.6)$ & $19(30.2)$ & 0.1 & $0.0-1.0$ \\
\hline \multicolumn{5}{|l|}{ Toilet use location } \\
\hline 1st floor male restroom & $6(35.3)$ & $28(47.5)$ & 0.6 & $0.2-2.1$ \\
\hline 1st floor female restroom & $10(58.8)$ & $11(18.6)$ & 6.0 & $1.7-23.6$ \\
\hline 2nd floor male restroom & $4(23.5)$ & $35(59.3)$ & 0.2 & $0.0-0.8$ \\
\hline 2nd floor female restroom & $12(70.6)$ & $16(27.1)$ & 6.3 & $1.7-26.5$ \\
\hline 3rd floor male restroom & $5(29.4)$ & $25(42.4)$ & 0.6 & $0.1-2.0$ \\
\hline \multicolumn{5}{|l|}{ Toilet cleaned at } \\
\hline 1st floor male restroom & $1(5.9)$ & $11(18.6)$ & 0.3 & $0.0-2.2$ \\
\hline 1st floor female restroom & $10(58.8)$ & $4(6.8)$ & 18.0 & $4.0-104.4$ \\
\hline \multicolumn{5}{|l|}{ Bathing } \\
\hline At Facility C & $10(58.8)$ & $43(72.9)$ & 0.5 & $0.2-2.0$ \\
\hline Bathed in a bathtub & $10(58.8)$ & $30(50.8)$ & 2.6 & $0.5-27.6$ \\
\hline Frequently drank the hot water when bathing & $1(5.9)$ & $6(10.2)$ & 0.4 & $0.0-4.1$ \\
\hline
\end{tabular}

$\mathrm{Cl}=$ confidence interval, $\mathrm{OR}=$ odds ratio

* The day care groups A1 and A2 consisted of mainly residents of Facility A; B1 and B2 consisted mainly of those of Facility B; S consisted of those who were on occupational therapy; and $\mathrm{V}$ consisted of those who need more attention of the staff.

** Not all individuals provided responses, so the number of responses may not be the total numbers of cases and controls.

The risk factors with statistically significant odds ratios are emphasized with bold font. 
Table 3. Individual characteristics associated with EHEC 0157:H7 infection among individuals not able to independently wash their hands during an outbreak among residents of Facilities $A$ and $B$ at an institute for people with intellectual disabilities, Japan, 2005 (34 cases and 82 controls)

\begin{tabular}{|c|c|c|c|c|}
\hline \multirow{3}{*}{ Stratified risk factors } & \multicolumn{2}{|c|}{ Unable to wash hands independently } & \multirow{3}{*}{ OR } & \multirow{3}{*}{$95 \% \mathrm{Cl}$} \\
\hline & & & & \\
\hline & Cases (\%) & Controls (\%) & & \\
\hline Overall & 31 (91.2) & $54(65.9)$ & 5.3 & $1.5-29.4$ \\
\hline \multicolumn{5}{|l|}{ Sex } \\
\hline Male & 25 (96.2) & $40(65.6)$ & 12.9 & $1.8-562.6$ \\
\hline Female & $6(75.0)$ & $14(66.7)$ & 1.5 & $0.2-18.7$ \\
\hline M-H adjusted & - & - & 5.3 & $1.5-18.7$ \\
\hline \multicolumn{5}{|l|}{ Allotriophagic behaviour } \\
\hline Yes & $10(100.0)$ & $9(100.0)$ & - & - \\
\hline No & $21(87.5)$ & $45(61.6)$ & 4.3 & $1.1-24.6$ \\
\hline M-H adjusted & - & - & 4.4 & $1.2-16.0$ \\
\hline \multicolumn{5}{|c|}{ Needs assistance in defecation } \\
\hline Yes & $16(100.0)$ & $20(95.2)$ & - & - \\
\hline No & $15(83.3)$ & $34(55.7)$ & 3.9 & $1.0-23.2$ \\
\hline M-H adjusted & - & - & 4.3 & $1.1-16.3$ \\
\hline
\end{tabular}

$\mathrm{Cl}=$ confidence interval, $\mathrm{OR}=$ odds ratio, $\mathrm{M}-\mathrm{H}=$ Mantel-Haenszel method

The items with statistically significant odds ratios are emphasized with bold font.

intellectual disabilities. The source of the outbreak could not be identified; however, the infection may have spread through the environment contaminated with EHEC. The residents who cleaned a particular restroom at Facility $C$ had 18 times the odds of testing positive for EHEC compared to those who did not, and neither samples from meal remnants nor stool samples from staff who worked in the kitchen yielded EHEC. At Facilities $A$ and $B$, it is likely that the infection spread via person-to-person contact because those who were unable to wash their own hands were more at risk. Environmental contamination was also supported by the findings that no single peak in the epidemic curve was noted, no episodes were reported in which a possible single source of infection was suspected and limitations in personal and toilet hygiene were confirmed. EHEC spread through contaminated environments has been previously reported; ${ }^{7}$ thus our findings are consistent with previous reports.

Infection spread via person-to-person contact is the leading cause of most EHEC outbreaks in institutional settings in Japan and elsewhere, particularly at day cares, schools ${ }^{13-16}$ and homes for older people. ${ }^{17}$ Foodborne infections ${ }^{18}$ and infection spread through the environment were sometimes suspected but were not supported by analytic epidemiology. ${ }^{13}$ Thus, this study is unique in that cleaning a certain restroom was implicated by analytical epidemiology as a possible common source.

In this outbreak, about two thirds of cases were asymptomatic. In Japan, active case-finding routinely includes testing asymptomatic contacts. ${ }^{14,19}$ According to the national surveillance data, one third of EHEC cases in Japan were asymptomatic. ${ }^{20}$ Over three fourths of the cases in our setting were adults, supporting a previous report that the proportion of cases that were symptomatic declined with age. ${ }^{14}$ Additionally, the doses of EHEC bacilli were likely small and thus not everyone developed symptoms. Asymptomatic carriers or recovered patients may shed EHEC for more than 30 days; ${ }^{21}$ however, humans are not considered as reservoirs. ${ }^{22}$ During an outbreak in Australia in 2007, an asymptomatic sibling spread EHEC to another sibling who developed HUS. ${ }^{23}$ The role of asymptomatic carriers of EHEC in outbreaks should not be underestimated.

Our study has both strengths and limitations. Since all the residents and staff members of the institution were 
tested for EHEC in their stools, we were able to identify infections that were asymptomatic. Although we believe most infections were transmitted through the environment at Facility $\mathrm{C}$, the environmental specimens did not yield the pathogen, most probably because the environment, particularly the door knobs, floor and tables, were disinfected shortly before the environmental samples were collected for bacteriological tests. In addition, residents with intellectual disabilities may have limited ability to provide comprehensive behavioural or risk information, and thus recall and information biases are likely. To minimize these biases, we verified the participants' responses with staff members' records.

We recommend that in institutional settings, particularly those that accommodate people with intellectual disabilities, staff should pay close attention to personal and toilet hygiene of the residents, and restrooms should be cleaned as often as possible to reduce possible infection via contact with contaminated surfaces. The infective dose of EHEC is small (lower than 700 organisms). ${ }^{24}$ Institutions should also have a symptomatic surveillance system and monitor trends in diarrhoea incidence among residents. Prefectural governments should strengthen their surveillance systems, including pathogen surveillance with routine PFGE tests, to detect potential outbreaks involving multiple prefectures. Local health offices should provide congregate settings, including health facilities, with training about communicable diseases to prevent outbreaks.

\section{Acknowledgements}

The authors would like to thank the staff members of the Health Division, Welfare and Health Department, Oita Prefecture, Usuki Health Office, Oita Prefecture Hygiene and Environment Centre and the Field Epidemiology Training Programme (FETP), National Institute of Infectious Disease, Tokyo, Japan, for their kind support of our investigation.

\section{Funding}

This investigation was funded by a grant from the Ministry of Health, Labour, and Welfare, Japan.

\section{Conflict of interest}

None declared.

\section{References}

1. Riley LW, Remis RS, Helgerson SD, McGee HB, Wells JG, Davis BR, et al. Hemorrhagic colitis associated with a rare Escherichia coli serotype. N Engl J Med. 1983 Mar 24;308(12):681-5. doi:10.1056/ NEJM198303243081203 pmid:6338386

2. Bell BP, Goldoft M, Griffin PM, Davis MA, Gordon DC, Tarr PI, et al. A multistate outbreak of Escherichia coli 0157:H7-associated bloody diarrhea and hemolytic uremic syndrome from hamburgers. The Washington experience. JAMA. 1994 Nov 2;272(17):1349-53. doi:10.1001/jama.1994.03520170059036 pmid:7933395

3. Bruneau A, Rodrigue $H$, Ismäel J, Dion R, Allard R. Outbreak of $\mathrm{E}$. coli $0157: \mathrm{H} 7$ associated with bathing at a public beach in the Montreal-Centre region. Can Commun Dis Rep. 2004 Aug 1;30(15):133-6. pmid:15315240

4. Carter AO, Borczyk AA, Carlson JA, Harvey B, Hockin JC, Karmali $\mathrm{MA}$, et al. A severe outbreak of Escherichia coli 0157:H7-associated hemorrhagic colitis in a nursing home. N Engl J Med. 1987 Dec 10;317(24):1496-500. doi:10.1056/NEJM198712103172403 pmid:3317047

5. Centers for Disease Control and Prevention (CDC). Outbreaks of Escherichia coli 0157:H7 associated with petting zoos-North Carolina, Florida, and Arizona, 2004 and 2005. MMWR Morb Mortal Wkly Rep. 2005 Dec 23;54(50):1277-80. pmid:16371942

6. Muto T, Matsumoto Y, Yamada M, Ishiguro Y, Kitazume H, Sasaki $\mathrm{K}$, et al. Outbreaks of enterohemorrhagic Escherichia coli 0157 infections among children with animal contact at a dairy farm in Yokohama City, Japan. Jpn J Infect Dis. 2008 Mar;61(2):161-2. pmid:18362413

7. Varma JK, Greene KD, Reller ME, DeLong SM, Trottier J, Nowicki SF, et al. An outbreak of Escherichia coli 0157 infection following exposure to a contaminated building. JAMA. 2003 Nov 26;290(20):2709-12. doi:10.1001/jama.290.20.2709 pmid:14645313

8. Michino H, Araki K, Minami S, Takaya S, Sakai N, Miyazaki M, et al. Massive outbreak of Escherichia coli 0157:H7 infection in schoolchildren in Sakai City, Japan, associated with consumption of white radish sprouts. Am J Epidemiol. 1999 Oct 15;150(8):787-96. doi:10.1093/oxfordjournals.aje.a010082 pmid:10522649

9. Oita Prefectural Institute of Health and Environment (formerly known as Oita Prefecture Hygiene and Environment Centre) [website]. Oita: Oita Prefectural Government; 2015 (http://www.pref. oita.jp.e.ro.hp.transer.com/site/13002/, accessed on 7 February 2019).

10. Manual for examination and diagnosis of enterohemorrhagic Escherichia coli (EHEC). Tokyo: National Institute of Infectious Diseases; $2017 \quad$ (https://www.niid.go.jp/niid/images/lab-manual/ EHEC20170215.pdf, accessed on 7 March 2018).

11. Terajima J, Izumiya H, Iyoda S, Tamura K, Watanabe H. High genomic diversity of enterohemorrhagic Escherichia coli isolates in Japan and its applicability for the detection of diffuse outbreak. Jpn J Infect Dis. 2002 Feb;55(1):19-22. pmid:11971157

12. Katz S. Assessing self-maintenance: activities of daily living, mobility, and instrumental activities of daily living. J Am Geriatr Soc. 1983 Dec;31(12):721-7. doi:10.1111/j.1532-5415.1983.tb03391.x pmid:6418786

13. Bayliss L, Carr R, Edeghere O, Knapper E, Nye K, Harvey G, et al. School outbreak of Escherichia coli $\mathrm{O} 157$ with high levels of transmission, Staffordshire, England, February 2012. J Public Health (Oxf). 2016 Sep;38(3):e247-53. doi:10.1093/pubmed/fdv122 pmid:26364319

14. Kanayama A, Yahata Y, Arima Y, Takahashi T, Saitoh T, Kanou $\mathrm{K}$, et al. Enterohemorrhagic Escherichia coli outbreaks related 
to childcare facilities in Japan, 2010-2013. BMC Infect Dis 2015 Nov 20;15(1):539. doi:10.1186/s12879-015-1259-3 pmid:26589805

15. Sonoda C, Tagami A, Nagatomo D, Yamada S, Fuchiwaki R, Haruyama M, et al. An enterohemorrhagic Escherichia coli 026 outbreak at a nursery school in Miyazaki, Japan. Jpn J Infect Dis. 2008 Jan;61(1):92-3. pmid:18219147

16. Gouveia S, Proctor ME, Lee MS, Luchansky JB, Kaspar CW. Genomic comparisons and Shiga toxin production among Escherichia coli 0157:H7 isolates from a day care center outbreak and sporadic cases in southeastern Wisconsin. J Clin Microbiol. 1998 Mar;36(3):727-33. pmid:9508303

17. Afza M, Hawker J, Thurston H, Gunn K, Orendi J. An outbreak of Escherichia coli 0157 gastroenteritis in a care home for the elderly. Epidemiol Infect. 2006 Dec;134(6):1276-81. doi:10.1017/ S0950268806006546 pmid:16740198

18. Preston M, Borczyk A, Davidson R, McGeer A, Bertoli J, Harris S, et al. Hospital outbreak of Escherichia coli 0157:H7 associated with rare phage type - Ontario. Canadian communicable disease report. 1997;23-05:33-37 (https://pdfs.semanticscholar.org/5972/ bd18b94cab06f455754e755e8e89a5cc4c4b.pdf, accessed on 7 February 2019).

19. Yamazaki S, et al. Kansensho yobou hikkei (A handbook for prevention of communicable disease). Tokyo, Japan: Nihon Koshu Eisei Kyokai; 1999. pp. 210-3 (in Japanese).
20. National Institute of Infectious Diseases. Enterohemorrhagic Escherichia coli (EHEC) infection, as of April 2017. Japan Infectious Agent Surveillance Report. 2017;38:87-8 (https://www.niid.go.jp/ niid/en/basic-science/865-iasr/7282-447te.html, accessed on 7 February 2019).

21. Swerdlow DL, Griffin PM. Duration of faecal shedding of Escherichia coli 0157:H7 among children in day-care centres. Lancet. 1997 Mar 15;349(9054):745-6. doi:10.1016/S0140-6736(05)601961 pmid:9074570

22. Beutin L, Martin A. Outbreak of shiga toxin-producing Escherichia coli (STEC) 0104:H4 infection in Germany causes a paradigm shift with regard to human pathogenicity of STEC strains. J Food Prot. 2012 Feb;75(2):408-18. doi:10.4315/0362-028X.JFP-11-452 pmid:22289607

23. Hanna JN, Humphreys JL, Ashton SE, Murphy DM. Haemolytic uraemic syndrome associated with a family cluster of enterohaemorrhagic Escherichia coli. Commun Dis Intell Q Rep. 2007 Sep;31(3):300-3. pmid:17974224

24. Tuttle J, Gomez T, Doyle MP, Wells JG, Zhao T, Tauxe RV, et al. Lessons from a large outbreak of Escherichia coli 0157:H7 infections: insights into the infectious dose and method of widespread contamination of hamburger patties. Epidemiol Infect. 1999 Apr;122(2):185-92. doi:10.1017/S0950268898001976 pmid:10355781 